\title{
Selective Inhibition of Striatal Fast-Spiking Interneurons Causes Dyskinesias
}

\author{
Aryn H. Gittis, ${ }^{1}$ Daniel K. Leventhal, ${ }^{2}$ Benjamin A. Fensterheim, ${ }^{3}$ Jeffrey R. Pettibone, ${ }^{3}$ Joshua D. Berke, ${ }^{3,4,5}$ \\ and Anatol C. Kreitzer ${ }^{1,6,7}$ \\ ${ }^{1}$ Gladstone Institute of Neurological Disease, San Francisco, California 94158, ${ }^{2}$ Department of Neurology, ${ }^{3}$ Department of Psychology, ${ }^{4}$ Movement \\ Disorders Program, and ${ }^{5}$ Neuroscience Program, University of Michigan, Ann Arbor, Michigan 48109, and ${ }^{6}$ Department of Physiology, and ${ }^{7}$ Department of \\ Neurology, University of California, San Francisco, San Francisco, California 94158
}

Fast-spiking interneurons (FSIs) can exert powerful control over striatal output, and deficits in this cell population have been observed in human patients with Tourette syndrome and rodent models of dystonia. However, a direct experimental test of striatal FSI involvement in motor control has never been performed. We applied a novel pharmacological approach to examine the behavioral consequences of selective FSI suppression in mouse striatum. IEM-1460, an inhibitor of GluA2-lacking AMPARs, selectively blocked synaptic excitation of FSIs but not striatal projection neurons. Infusion of IEM-1460 into the sensorimotor striatum reduced the firing rate of FSIs but not other cell populations, and elicited robust dystonia-like impairments. These results provide direct evidence that hypofunction of striatal FSIs can produce movement abnormalities, and suggest that they may represent a novel therapeutic target for the treatment of hyperkinetic movement disorders.

\section{Introduction}

Dystonia, tics, and chorea are symptoms of hyperkinetic movement disorders that are thought to involve impaired GABAergic function, most notably within striatum (Levy and Hallett, 2002; Kalanithi et al., 2005; Kumar et al., 2010). In experimental animals, interfering with striatal GABAergic networks via local infusion of $\mathrm{GABA}_{\mathrm{A}}$ antagonists elicits tic-like movements and other dyskinesias (McCairn et al., 2009; Worbe et al., 2009). However, determining how such behavioral effects arise is a challenge, as information processing within striatal microcircuits involves multiple distinct forms of GABAergic signaling that are difficult to disentangle. First, medium spiny projection neurons (MSNs) communicate with each other using synapses predominantly onto distal dendrites (Wilson, 2007). These connections are typically nonreciprocal and individually weak (Gustafson et al., 2006; Taverna et al., 2008), but in aggregate may underlie essential competitive dynamics between striatal cell assemblies (Humphries et al., 2009; Ponzi and Wickens, 2010). Second, fastspiking interneurons (FSIs) can strongly influence MSN spike timing (Koos and Tepper, 1999; Gittis et al., 2010) via perisomatic synapses (Bennett and Bolam, 1994). Further sources of GABAergic signaling in the striatum include persistent low-

Received July 28, 2011; revised Aug. 30, 2011; accepted Sept. 17, 2011.

Author contributions: A.H.G., D.K.L., J.D.B., and A.C.K. designed research; A.H.G., D.K.L., B.A.F., and J.R.P. performed research; A.H.G., D.K.L., and J.D.B. analyzed data; A.H.G., D.K.L., J.D.B., and A.C.K. wrote the paper.

This work was supported by the Tourette Syndrome Association, the Bachmann-Strauss Dystonia and Parkinson's Fund, the Dystonia Medical Research Foundation, NIH Grants R01 NS064984 (to A.C.K.) and F32 NS065641 (to A.H.G.), the Pew Biomedical Scholars Program, the W.M. Keck Foundation, and the McKnight Foundation. We thank A. Nelson and A. Kravitz for help with pilot experiments, and Vaughn Hetrick for technical assistance.

Correspondence should be addressed to either Joshua D. Berke or Anatol C. Kreitzer at the above addresses. E-mail: jdberke@umich.edu or akreitzer@gladstone.ucsf.edu.

DOI:10.1523/JNEUROSCI.3875-11.2011

Copyright $\odot 2011$ the authors $\quad 0270-6474 / 11 / 3115727-05 \$ 15.00 / 0$ threshold spiking (PLTS) interneurons and external GABAergic input from the globus pallidus (Bevan et al., 1998). There is evidence that the FSI-mediated component of striatal GABA signaling is central to at least some hyperkinetic phenomena-reduced numbers of FSIs have been seen in human Tourette syndrome (Kalanithi et al., 2005) and animal models of dystonia (Gernert et al., 2000). Yet this hypothesis has never been subject to direct experimental test.

MSNs account for $\sim 95 \%$ of neurons in the striatum, with the other major cell types consisting of FSIs, PLTS GABAergic interneurons, and cholinergic interneurons. PLTS and cholinergic interneurons are spontaneously active due to intrinsic pacemaking currents. By contrast, FSIs and MSNs require synaptic input to reach spike threshold, so blocking glutamate receptors suppresses spiking. Here, we present a novel pharmacological approach to rapidly and selectively reduce FSI firing rates in the awake striatum, taking advantage of the fact that FSIs, but not MSNs, express GluA2-lacking AMPARs. We show that reducing FSI-mediated GABAergic signaling within striatum is sufficient to induce abnormal involuntary movements that resemble human dystonia.

\section{Materials and Methods}

Slice electrophysiological recordings. Coronal sections (300 $\mu \mathrm{m}$ thick) containing dorsolateral striatum were prepared from brains of 3- to 5-weekold male and female Lhx6-EGFP mice on an FVB genetic background. Sections were prepared on a Leica VT1000S vibratome in an ice-cold sucrose cutting solution containing the following (in $\mathrm{mm}$ ): $79 \mathrm{NaCl}, 23$ $\mathrm{NaHCO}_{3}, 68$ sucrose, 12 glucose, $2.3 \mathrm{KCl}, 1.1 \mathrm{NaH}_{2} \mathrm{PO}_{4}, 6 \mathrm{MgCl}_{2}$, and 0.5 $\mathrm{CaCl}_{2}$. Sections were bisected to generate left and right hemisphere slices and transferred to a chamber filled with warmed carbogenated ACSF containing the following (in $\mathrm{mm}$ ): $125 \mathrm{NaCl}, 26 \mathrm{NaHCO}_{3}, 2.5 \mathrm{KCl}, 1$ $\mathrm{MgCl}_{2}, 2 \mathrm{CaCl}_{2}, 1.25 \mathrm{NaH}_{2} \mathrm{PO}_{4}$, and 12.5 glucose. 
A

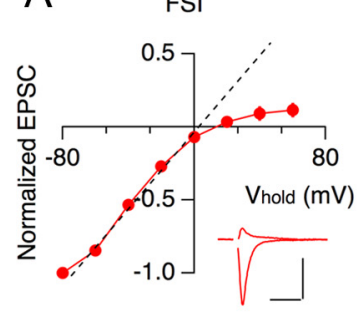

C

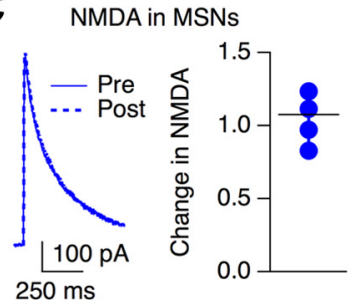

MSN

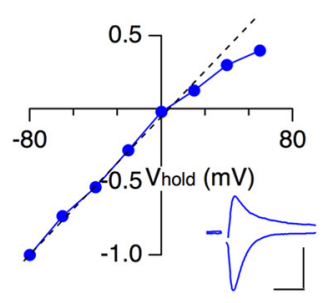

D

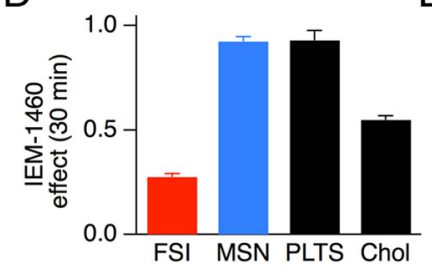

B

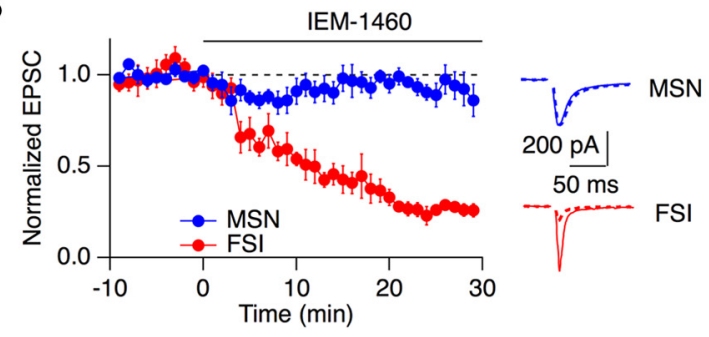

$\mathrm{E}$

Cholinergic

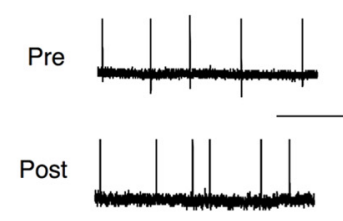

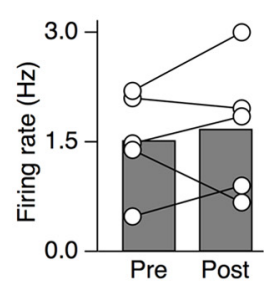

Figure 1. IEM-1460 reduces EPSCs in FSIs but not MSNs. $A, I-V$ curves of AMPAR-mediated currents in FSIs $(n=5)$ or MSNs $(n=5)$. Rectification index (current at $+60 \mathrm{mV} /$ current at $-60 \mathrm{mV}$ ) was $0.11 \pm 0.11$ for FSIs and $0.54 \pm 0.08$ for MSNs. Insets show representative AMPAR-mediated currents from an FSI (red) and an MSN (blue) at $V_{\text {hold }}=-80 \mathrm{mV}$ (inward current) and $+60 \mathrm{mV}$ (outward current). Stimulus artifacts were removed for clarity. Scale bar (both insets): vertical, 400 pA; horizontal, $10 \mathrm{~ms}$. B, IEM-1460 (50 $\mu$ M) reduces EPSC amplitudes in FSIs ( $n=6$ ), but not MSNs $(n=6)$. Insets show EPSCs recorded from a representative MSN (top) and FSI (bottom); solid line is the average trace $0-5$ min before IEM-1460; dotted line is the average trace $25-30$ min after IEM-1460. EPSCs were measured every $20 \mathrm{~s}$ and binned in $1 \mathrm{~min}$ bins. EPSCS were normalized by the average amplitude recorded during a $10 \mathrm{~min}$ baseline before drug application (-10 to 0 min). C, IEM-1460 $(50 \mu \mathrm{M})$ does not affect NMDAR-mediated EPSCs in MSNs ( $V_{\text {hold }}=+40 \mathrm{mV}$ ). Left, Example of NMDA-mediated currents recorded from an MSN $0-5$ min before (Pre; solid line) and 25-30 min after (Post; dotted line) bath application of IEM-1460. Right, Average change in NMDA-mediated currents in MSNs after 30 min of IEM-1460 $(n=4)$. $D$, Summary of the change in AMPAR-mediated EPSCs by IEM-1460 (EPSC amplitudes $25-30$ min after IEM-1460 application/EPSC amplitudes $0-5$ min before IEM-1460 application) in each cell type. E, Left, Spontaneous, extracellularly recorded action potentials from a representative cholinergic (Chol) interneuron in slice $0-5$ min before and $25-30$ min after IEM-1460 application. Scale bar: horizontal, $1 \mathrm{~s}$; vertical, $1 \mathrm{mV}$. Right, Change in spontaneous firing rate of cholinergic interneurons recorded after IEM-1460 application. Bars represent the average and circles are individual cells.

All recordings were performed at $31-33^{\circ} \mathrm{C}$ in ACSF (see above) in the presence of $50 \mu \mathrm{M}$ picrotoxin to block $\mathrm{GABA}_{\mathrm{A}}$-mediated currents. For experiments where rectification of AMPAR-mediated EPSCs was measured, $50 \mu \mathrm{M}$ APV was also included in the external medium. To isolate NMDAR-mediated EPSCs in MSNs (Fig. 1C), neurons were voltage clamped at $+40 \mathrm{mV}$ and $5 \mu \mathrm{M}$ NBQX was included in the bath to block AMPAR-mediated currents. In most experiments, EPSCs were evoked using a glass stimulating electrode positioned in the striatum within $100-200 \mu \mathrm{M}$ of the recorded neuron. The tissue was stimulated with 0.2 ms electrical pulses every $20 \mathrm{~s}(2-6 \mu \mathrm{A}$ for FSIs and MSNs and $10-20 \mu \mathrm{A}$ for PLTS). Because stronger stimuli were required to elicit EPSCs onto cholinergic interneurons, a concentric bipolar stimulating electrode was used for these experiments. The stimulating electrode was positioned within the striatum $>500 \mu \mathrm{m}$ away from the recorded neuron. The tissue was stimulated with $0.2 \mathrm{~ms}$ electrical pulses every $20 \mathrm{~s}(10-20 \mathrm{~mA})$.

Internal solution contained the following (in mM): $120 \mathrm{CsMeSO}_{3}, 15$ CsCl, $8 \mathrm{NaCl}$, 0.5 EGTA, 10 HEPES, 2 Mg-ATP, 0.3 Na-GTP, 5 QX-314, $\mathrm{pH}$ 7.3. For experiments measuring NMDA currents in MSNs, we added $10 \mathrm{~mm}$ BAPTA; and for experiments measuring rectification, we added both $10 \mathrm{~mm}$ BAPTA and $0.1 \mathrm{~mm}$ spermine.

Data were collected with a MultiClamp 700B amplifier (Molecular Devices) and ITC-18 A/D board (HEKA) using Igor Pro software (Wavemetrics) and custom acquisition routines (mafPC, courtesy of M. A. Xu-Friedman, University of Buffalo, Buffalo, NY). Recordings were filtered at $2 \mathrm{kHz}$ and digitized at $10 \mathrm{kHz}$. Electrodes were made from borosilicate glass (pipette resistance, 2-4 M $\Omega$ ). During recordings, $R_{\text {series }}$ was monitored and recordings were terminated if $R_{\text {series }}$ changed by $>30 \%$.

FSIs and PLTS interneurons were identified using GFP fluorescence and distinguished using $R_{\text {in }}$ and $I_{\text {hold }}$ in whole-cell recordings, as previously established (Gittis et al., 2010). Cholinergic interneurons were identified by their large somata and lack of GFP fluorescence in the Lhx6-EGFP line.

In vivo electrophysiological recordings. Both male and female mice were used for in vivo experiments. Two mice were implanted with 64-channel silicon probes $(8 \times 8$ grid, $200 \mu \mathrm{m}$ spacing; NeuroNexus Technologies) with the long axis of the probe aligned at $25^{\circ}$ from the anterior-posterior direction so that all contacts were in left dorsal/lateral striatum. Infusion cannulae (Plastics One) had their tips near the center of the probe grids (tip: AP $+0.4 \mathrm{~mm}, \mathrm{ML} \pm 2.2 \mathrm{~mm}$, DV $2.5 \mathrm{~mm}$ ). Following recovery from surgery, wideband data $(1-9000 \mathrm{~Hz})$ were recorded continuously from all sites at 31,250 Hz, as described previously (Wiltschko et al., 2010). Signals were referenced to a skull screw over the midline cerebellum. During each session, a $15 \mathrm{~min}$ baseline recording was followed by infusion of ACSF $(0.25 \mu \mathrm{l} / \mathrm{min}$ for $2 \mathrm{~min}$, with the cannula left in place for 2 min postinfusion). After $30 \mathrm{~min}, 1 \mathrm{~mm} \mathrm{IEM-1460} \mathrm{dissolved} \mathrm{in} \mathrm{ACSF} \mathrm{was}$ infused using the same parameters. The recording continued for at least $90 \mathrm{~min}$.

To extract single unit activity, the wideband signals were waveletfiltered offline using custom Matlab scripts (Wiltschko et al., 2008) and spikes were clustered in Offline Sorter (Plexon). The resulting spike timestamps were used to extract mean wideband waveforms for each unit. To classify units as FSIs or MSNs (Berke et al., 2004; Gage et al., 2010), the full width at half-maximum (FWHM) and peak-to-valley (P-V) times were used (FSI: FWHM, 50-150 ms; P-V, 50-455 ms; MSN: FWHM, $150-450 \mathrm{~ms}, \mathrm{P}-\mathrm{V}, 560-1500 \mathrm{~ms}$; Fig. 2 B). None of the cells classified as MSNs or FSIs had the regular tonic firing characteristic of cholinergic interneurons. Any units that did not meet these waveform criteria were labeled as "unclassified." Inverted units (units with a more prominent positive than negative waveform) were automatically labeled as unclassified, as the FWHM and P-V times have no clear meaning for these units.

Behavioral experiments. Three groups of mice were implanted with infusion cannulae. For each group, half of the implants were in the right hemisphere. For the first group (Group 1, $n=6$; Fig. 3, black circles), the target was dorsolateral striatum (AP $+0.4 \mathrm{~mm}, \mathrm{ML} \pm 2.4 \mathrm{~mm}, \mathrm{DV} 2.8$ $\mathrm{mm}$ relative to bregma). After $2 \mathrm{~d}$ for recovery, the mice were infused daily with one of three concentrations $(0.25 \mathrm{~mm}, 1 \mathrm{~mm}$, or $2.5 \mathrm{~mm})$ of IEM-1460 dissolved in ACSF or with ACSF alone. Doses were delivered in a counterbalanced order over four successive days.

The second group (Group 2, $n=4$; Fig. 3, gray circles) was implanted with cannulae targeted to the same coordinates, but received combined infusions of mecamylamine (a nicotinic receptor antagonist) and scopolamine (a nonspecific muscarinic antagonist). Two doses were used: $5 \mathrm{mg}$ of mecamylamine with $10 \mathrm{mg}$ of scopolamine, and $10 \mathrm{mg}$ of mecamyl- 

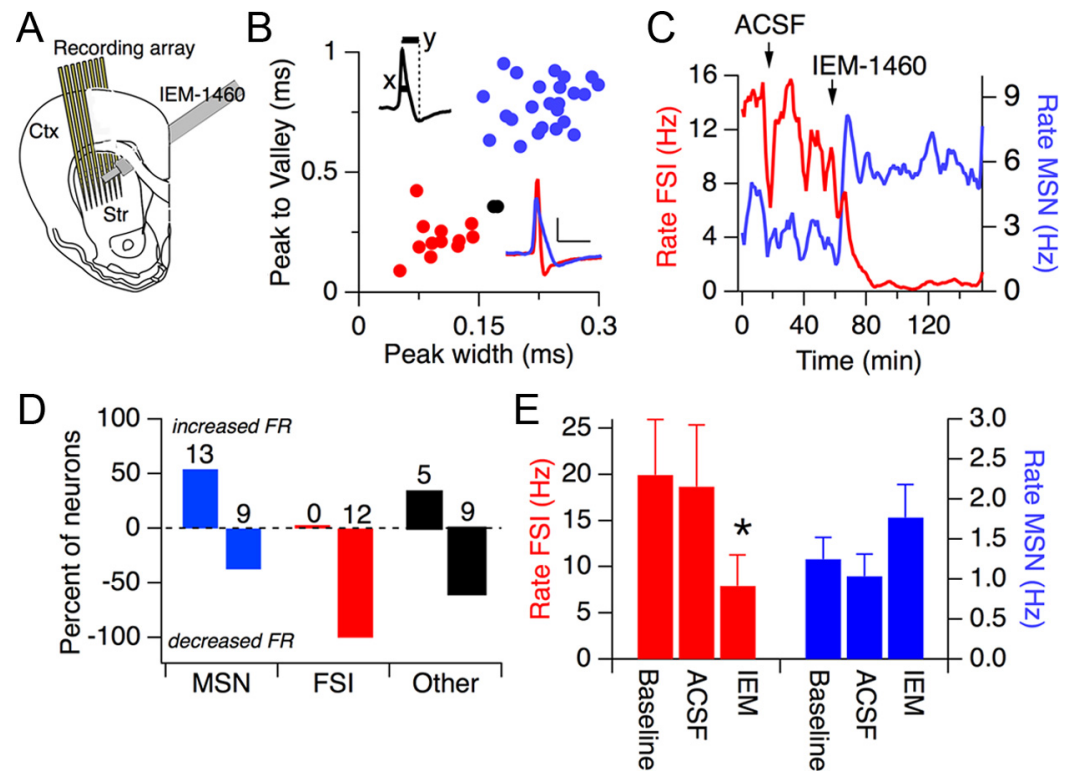

Figure 2. IEM-1460 selectively reduces FSI activity in vivo. A, Schematic of in vivo recording configuration and drug infusion. An $8 \times 8$ chronic recording array was placed in dorsolateral striatum with an infusion cannula angled toward the array. Ctx, Cortex; Str, striatum. $\boldsymbol{B}$, Scatter plot of mean spike waveform parameters used to classify FSIs (red) and MSNs (blue). Cells were classified based on the width at half-height $(x)$ and peak-to-valley time ( $y$ ). Cells that could not clearly be sorted as FSI or MSNs were called unclassified (black; cells with inverted waveforms not shown). Bottom right, Inset, Examples of extracellular waveforms (negative voltage upward) recorded from a presumed FSI (red) and presumed MSN (blue). Scale bar: vertical, $0.1 \mathrm{mV}$; horizontal, $1 \mathrm{~ms}$. C, Example of firing rates recorded simultaneously from an FSI and MSN during infusion of ACSF and then IEM-1460 (1 mM). IEM-1460 strongly suppressed spiking of the FSI and slightly increased spiking of this MSN. D, Summary of firing rate (FR) changes observed for all FSIs, MSNs, and unclassified neurons after IEM-1460 infusion. Number of neurons is listed above each bar [54\% (13/24) of MSNs increased their firing rates; $38 \%$ (9/24) decreased their firing rates; $8 \%$ (2/24) showed no change; $100 \%$ of FSIs (12/12) decreased their firing rates; $33 \%$ (5/15) of unclassified neurons increased their firing rates, $60 \%(9 / 15)$ decreased their firing rates, $7 \%(1 / 15)$ showed no change]. $E$, Average firing rates for the population of FSIs $(n=12)$ and MSNs $(n=24)$, after ACSF or IEM-1460 infusion. Note distinct $y$-axis scales for FSIs and MSNs. ACSF infusion did not significantly affect the firing rates of either population, whereas IEM-1460 significantly decreased the firing rates of FSIs, but not MSNs. ${ }^{*} p<0.01$ compared to both baseline and ACSF. Error bars show SEM.

amine with $20 \mathrm{mg}$ of scopolamine. These values were chosen to be on the high end of doses previously found to cause learning impairments in rats and mice (Schildein et al., 2002; Klinkenberg and Blokland, 2010).

The third group (Group 3, $n=4$; Fig. 3, open circles) was implanted with cannulae in dorsomedial striatum (AP $+0.4 \mathrm{~mm}, \mathrm{ML} \pm 1.25 \mathrm{~mm}$, DV $2.4 \mathrm{~mm}$ relative to bregma), and received infusions of ACSF and 2.5 mM IEM-1460 in counterbalanced order.

All drug infusions were performed at a rate of $0.25 \mu \mathrm{l} / \mathrm{min}$ for $2 \mathrm{~min}$. The infusion cannula was left in place for a further 2 min to allow drug diffusion, followed by 90 min of video recording. A clinical neurologist (D.K.L.) blind to the treatments scored $60 \mathrm{~s}$ segments every $5 \mathrm{~min}$ for the first $30 \mathrm{~min}$ after the infusion, and for every $10 \mathrm{~min}$ thereafter. Two previously described semiquantitative rating scales (Shirley et al., 2008) were used: a dyskinesia score that characterized the range of movement types and body parts involved, and an impairment score that characterized the severity of the movements. Briefly, 1 indicated minor motor impairment, 2 indicated moderate abnormalities with at most infrequent falls, 3 indicated significant impairment with frequent falls and severely impaired locomotion, and 4 indicated severe impairment with almost no ambulation (Shirley et al., 2008). The sole difference to the previously reported rating scales is that we also noted laterality for each relevant movement type (for example, neck twisting could be scored as ipsilateral or contralateral to the infusion, but neck flexion could not).

Data analysis and statistics. The area under each dyskinesia (or impairment) score versus time curve was calculated for each behavioral session. The areas under the curve (AUCs) in Group 1 at each dose were compared using ANOVA with post hoc Bonferroni corrections for pairwise comparisons. AUCs for Group 2 were analyzed in the same manner. AUCs at the $2.5 \mathrm{~mm}$ IEM dose for dorsomedial and dorsolateral striatum
(Groups 1 and 3) were compared with a twosample $t$ test. All tests were considered significant at $p<0.05$.

Single-unit firing rates were calculated for the period beginning 10 min after each infusion to $5 \mathrm{~min}$ before the next infusion (the baseline period began with the beginning of the recording; the IEM-1460 period ended with the end of the recording). The overall firing rate of each cell was computed as the number of spikes recorded in an epoch divided by the epoch duration. Firing rates of distinct cell populations in the baseline, ACSF, and IEM1460 epochs were compared using Friedman's test with post hoc pairwise comparisons (SPSS 18.0). To determine whether an individual unit changed its firing rate with each intervention, firing rates were computed in $1 \mathrm{~s}$ nonoverlapping windows during the ACSF and IEM-1460 epochs, creating a distribution of firing rates for each epoch. These rates were compared using paired $t$ tests, with $p<0.01$ considered to indicate a significant change in firing rate.

\section{Results}

As in many brain regions (McBain and Fisahn, 2001), glutamate receptors on striatal FSIs lack GluA2 subunits, as indicated by immunostaining (Bernard et al., 1997; Deng et al., 2007) and prominent inward rectification (Fig. $1 A$ ). In contrast, both physiological (Fig. $1 A, B$ ) and anatomical (Bernard et al., 1997; Deng et al., 2007) data suggest that MSN AMPARs contain GluA2 subunits. This difference in glutamate receptor composition enables the selective blockade of AMPARmediated EPSCs in FSIs $(-73 \pm 2 \%, n=$ 6) using dicationic adamantane derivatives such as IEM-1460 (Magazanik et al., 1997; Buldakova et al., 2007) (Fig. 1 B, D). IEM1460 did not block MSN AMPARs $(-8 \pm 13 \%, n=6$; Fig. $1 B, D)$, MSN NMDARs $(-1 \pm 9 \%, n=4$; Fig. $1 C)$, or PLTS AMPARs $(-7 \pm 23 \%, n=4$; Fig. $1 D)$. Even in the presence of $0.5 \mathrm{~mm}$ IEM-1460, EPSCs onto MSNs were not strongly affected ( $-12 \pm$ $3 \%, n=3)$. IEM-1460 also significantly reduced AMPARmediated currents in cholinergic interneurons $(-45 \pm 9 \%, n=$ 4; Fig. $1 D$ ), consistent with prior observations (Samoilova et al., 1999). However, the spontaneous firing rates of cholinergic cells were not affected (from $1.5 \pm 0.7 \mathrm{~Hz}$ before IEM-1460 application to $1.7 \pm 1.0 \mathrm{~Hz}$ after $20 \mathrm{~min}, n=5$; Fig. $1 E$ ).

To examine the effect of IEM-1460 on striatal neurons in vivo, we recorded from dorsolateral (sensorimotor) striatum in awake, unrestrained mice during local infusions of ACSF (control) or IEM-1460 (1 mM) (Fig. 1A). Presumed MSNs and FSIs were readily classified (Fig. $2 B$ ) using criteria previously established in rats (Berke, 2008; Gage et al., 2010; Wiltschko et al., 2010). IEM1460 consistently reduced the activity of all FSIs (12/12 cells; average rate of decrease: from 19.9 to $8.0 \mathrm{~Hz}, p=0.007$; Fig. $2 C-E)$. In contrast, MSNs showed no consistent response to IEM (Fig. 2C-E) and no significant change in average firing rate (from 1.2 to $1.8 \mathrm{~Hz}, p=0.33$ ).

Next, we analyzed the behavioral effects of intrastriatal IEM1460 over a range of doses. Mice receiving $1 \mathrm{~mm}$ IEM-1460 into dorsolateral striatum appeared relatively normal at rest. How- 
A

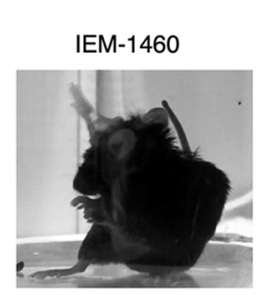

C
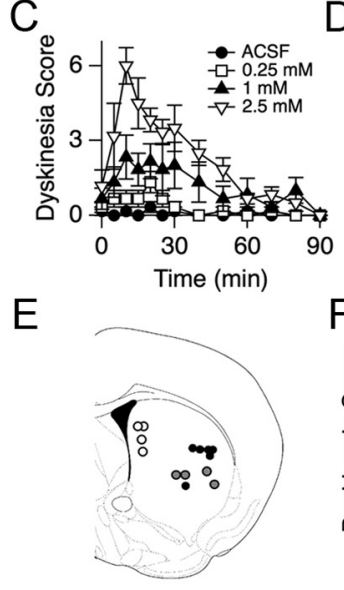

$\mathrm{F}$

G

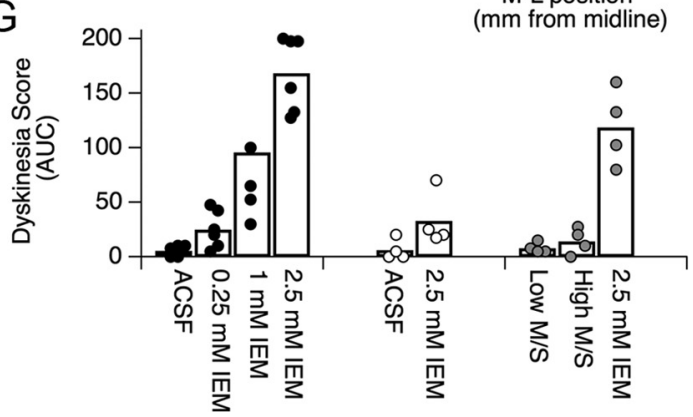

Figure 3. IEM-1460 causes hyperkinetic motor impairments in awake, unrestrained mice. $\boldsymbol{A}$, Picture of a mouse during a twisting episode after infusion of IEM-1460. Note the abnormal posture of the right (contralateral) arm and foot. $\boldsymbol{B}$, Average normalized dyskinesia score by body region, split by ipsilateral/contralateral side following infusion of $1 \mathrm{~mm}$ IEM-1460. C, Average dyskinesia score in $10 \mathrm{~min}$ bins observed during a $1.5 \mathrm{~h}$ period after infusion of ACSF for different concentrations of IEM-1460. D, Average impairment score after infusion of ACSF for different concentrations of IEM-1460. $E$, Schematic of striatum showing infusion sites for behavioral experiments. Black circles, Cannula placements for Group 1 (dorsolateral IEM-1460 infusions); gray circles, cannula placements for Group 2 (cholinergic antagonists then IEM-1460 infusions); white circles, cannula placements for Group 3 (dorsomedial IEM-1460 infusions). $\boldsymbol{F}$, Scatter plot showing strong correlation $\left(r^{2}=0.81, p=1.1 \times 10^{-5}\right)$ between the mediallateral position of the infusion cannula and the area under the dyskinesia score versus time curve (AUC) for all $2.5 \mathrm{~mm}$ IEM infusions. G, Average dyskinesia score AUC following infusion of ACSF, IEM-1460, or mecamylamine/scopolamine (M/S) (see Materials and Methods for doses) into dorsomedial or dorsolateral striatum. Circles indicate individual data points for each mouse.

ever, as they initiated activity, the mice developed prolonged, twisted postures resembling dystonia, together with jerky, repetitive movements (Fig. 3A). These abnormal movements were directed contralaterally and predominantly involved the head and neck (Fig. 3B). At the highest dose of IEM-1460 (2.5 mM), motor impairments became more continuous, widespread, and severe (Fig. 3C,D). In a separate group of mice, we infused this highest dose of IEM-1460 into dorsomedial striatum-a subregion that has few FSIs (Berke et al., 2004) —and observed only mild dyskinesia (Fig. 3E-G).

Although IEM-1460 also reduces excitatory synaptic input onto cholinergic interneurons (Fig. 1D), it is unlikely that the dyskinetic effects of IEM-1460 arise from a reduction in cholinergic signaling, as anticholinergics are used clinically to treat dystonia (Greene et al., 1988; Balash and Giladi, 2004), and many prior studies have found no acute behavioral impairment from intrastriatal cholinergic antagonism, ablation, or inactivation (Meana et al., 1992; Zhou et al., 1993; Witten et al., 2010). Nonetheless, to rule out this possibility, we infused a combination of the broad spectrum muscarinic antagonist scopolamine ( 10 or 20 $\mu \mathrm{g}$ ) and the nicotinic antagonist mecamylamine (5 or $10 \mu \mathrm{g}$ ) into the dorsolateral striatum. This combination did not produce substantial dyskinesias, even at doses much higher than are sufficient to produce learning impairments with intrastriatal infusion (M/S group; Fig. 3G) (Schildein et al., 2002; Klinkenberg and Blokland,

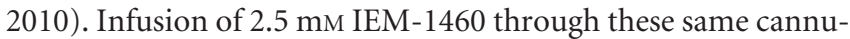
lae did produce significant motor impairment, as before (Fig. $3 G$ ). Together, these data strongly support the hypothesis that FSI inhibition is sufficient to produce hyperkinetic motor symptoms.

\section{Discussion}

Although FSIs comprise only $1 \%$ of striatal neurons, our results demonstrate that they are critical to the normal function of the striatum. We have shown that selective suppression of the FSImediated portion of striatal GABA transmission can produce a set of hyperkinetic motor impairments that resemble human dystonia. This observation has important implications for understanding the pathophysiology of movement disorders, as well as normal striatal physiology.

The modulation of circuit function with a pharmacological agent such as IEM-1460 has several advantages over viral and/or optogenetic strategies. First, transgenic mice are not required to achieve cell-type specificity, which enables IEM-1460 to be applied in unmodified animals of various species, including rats and primates. Second, IEM-1460 acts at every FSI in the injected region, whereas viral transduction rarely achieves $100 \%$ efficiency. Finally, IEM-1460 can achieve a sustained inhibition of FSIs without the need for prolonged light exposure, eliminating concerns about tissue heating.

Hyperkinetic movement disorders can be categorized as chorea, dystonia, myoclonus, or tics, with significant overlap between these phenotypes. Phenomenologically, striatal FSI suppression primarily led to dystonic postures. These results indicate that striatal FSI dysfunction may underlie some forms of human dystonia, with the caveat that specific physiologic deficits may not lead to identical motor manifestations in mice and humans. Given that striatal FSIs also show reduced firing rates following systemic injections of D2 antagonists (Wiltschko et al., 2010), these cells are now good candidates for involvement in primary dystonias, acute dystonic reactions to neuroleptics, and the off-period dystonia commonly observed in Parkinson disease.

Striatal FSIs innervate both direct- and indirect-pathway MSNs (Gittis et al., 2010; Planert et al., 2010) and are thought to provide powerful feedforward inhibition that shapes the firing patterns of MSNs (Koos et al., 2004; Mallet et al., 2005, 2006). Our results confirm that FSIs are critical nodes in striatal function. Even though we did not observe substantial changes in the average firing rate of the nearby MSN population following FSI suppression, MSNs form the sole output of the striatum to downstream basal ganglia nuclei. Thus, changes in MSN activitywhich MSNs are active and when--must ultimately be involved in the resulting abnormal behaviors. Our results are consistent with emerging findings that FSIs do not simply inhibit MSNs, but rather play a more subtle role in the organization of, and compe- 
tition between striatal projection cell assemblies (Berke, 2009; Humphries et al., 2009; Gage et al., 2010). Further defining how FSIs coordinate MSN activity patterns to shape striatal output will be an important goal for future large-scale recording studies (Carrillo-Reid et al., 2008), and selective drugs such as IEM-1460 are likely to be very useful tools for achieving this goal.

\section{Notes}

Supplemental material for this article is available at http://www.berkelab. org/Techniques.html. Video of a mouse after unilateral intrastriatal infusion of IEM-1460 showing dyskinesia and dystonia. This material has not been peer reviewed.

\section{References}

Balash Y, Giladi N (2004) Efficacy of pharmacological treatment of dystonia: evidence-based review including meta-analysis of the effect of botulinum toxin and other cure options. Eur J Neurol 11:361-370.

Bennett BD, Bolam JP (1994) Synaptic input and output of parvalbuminimmunoreactive neurons in the neostriatum of the rat. Neuroscience 62:707-719.

Berke JD (2008) Uncoordinated firing rate changes of striatal fast-spiking interneurons during behavioral task performance. J Neurosci 28:10075-10080.

Berke JD (2009) Fast oscillations in cortical-striatal networks switch frequency following rewarding events and stimulant drugs. Eur J Neurosci 30:848-859.

Berke JD, Okatan M, Skurski J, Eichenbaum HB (2004) Oscillatory entrainment of striatal neurons in freely moving rats. Neuron 43:883-896.

Bernard V, Somogyi P, Bolam JP (1997) Cellular, subcellular, and subsynaptic distribution of AMPA-type glutamate receptor subunits in the neostriatum of the rat. J Neurosci 17:819-833.

Bevan MD, Booth PA, Eaton SA, Bolam JP (1998) Selective innervation of neostriatal interneurons by a subclass of neuron in the globus pallidus of the rat. J Neurosci 18:9438-9452.

Buldakova SL, Kim KK, Tikhonov DB, Magazanik LG (2007) Selective blockade of $\mathrm{Ca} 2+$ permeable AMPA receptors in CA1 area of rat hippocampus. Neuroscience 144:88-99.

Carrillo-Reid L, Tecuapetla F, Tapia D, Hernández-Cruz A, Galarraga E, Drucker-Colin R, Bargas J (2008) Encoding network states by striatal cell assemblies. J Neurophysiol 99:1435-1450.

Deng YP, Xie JP, Wang HB, Lei WL, Chen Q, Reiner A (2007) Differential localization of the GluR1 and GluR2 subunits of the AMPA-type glutamate receptor among striatal neuron types in rats. J Chem Neuroanat 33:167-192.

Gage GJ, Stoetzner CR, Wiltschko AB, Berke JD (2010) Selective activation of striatal fast-spiking interneurons during choice execution. Neuron 67:466-479.

Gernert M, Hamann M, Bennay M, Löscher W, Richter A (2000) Deficit of striatal parvalbumin-reactive GABAergic interneurons and decreased basal ganglia output in a genetic rodent model of idiopathic paroxysmal dystonia. J Neurosci 20:7052-7058.

Gittis AH, Nelson AB, Thwin MT, Palop JJ, Kreitzer AC (2010) Distinct roles of GABAergic interneurons in the regulation of striatal output pathways. J Neurosci 30:2223-2234.

Greene P, Shale H, Fahn S (1988) Analysis of open-label trials in torsion dystonia using high dosages of anticholinergics and other drugs. Mov Disord 3:46-60.

Gustafson N, Gireesh-Dharmaraj E, Czubayko U, Blackwell KT, Plenz D (2006) A comparative voltage and current-clamp analysis of feedback and feedforward synaptic transmission in the striatal microcircuit in vitro. J Neurophysiol 95:737-752.

Humphries MD, Wood R, Gurney K (2009) Dopamine-modulated dynamic cell assemblies generated by the GABAergic striatal microcircuit. Neural Netw 22:1174-1188.

Kalanithi PS, Zheng W, Kataoka Y, DiFiglia M, Grantz H, Saper CB, Schwartz ML, Leckman JF, Vaccarino FM (2005) Altered parvalbumin-positive neuron distribution in basal ganglia of individuals with Tourette syndrome. Proc Natl Acad Sci U S A 102:13307-13312.
Klinkenberg I, Blokland A (2010) The validity of scopolamine as a pharmacological model for cognitive impairment: a review of animal behavioral studies. Neurosci Biobehav Rev 34:1307-1350.

Koos T, Tepper JM (1999) Inhibitory control of neostriatal projection neurons by GABAergic interneurons. Nat Neurosci 2:467-472.

Koos T, Tepper JM, Wilson CJ (2004) Comparison of IPSCs evoked by spiny and fast-spiking neurons in the neostriatum. J Neurosci 24:7916-7922.

Kumar P, Kalonia H, Kumar A (2010) Huntington's disease: pathogenesis to animal models. Pharmacol Rep 62:1-14.

Levy LM, Hallett M (2002) Impaired brain GABA in focal dystonia. Ann Neurol 51:93-101.

Magazanik LG, Buldakova SL, Samoilova MV, Gmiro VE, Mellor IR, Usherwood PN (1997) Block of open channels of recombinant AMPA receptors and native AMPA/kainate receptors by adamantane derivatives. J Physiol 505:655-663.

Mallet N, Le Moine C, Charpier S, Gonon F (2005) Feedforward inhibition of projection neurons by fast-spiking GABA interneurons in the rat striatum in vivo. J Neurosci 25:3857-3869.

Mallet N, Ballion B, Le Moine C, Gonon F (2006) Cortical inputs and GABA interneurons imbalance projection neurons in the striatum of parkinsonian rats. J Neurosci 26:3875-3884.

McBain CJ, Fisahn A (2001) Interneurons unbound. Nat Rev Neurosci 2:11-23.

McCairn KW, Bronfeld M, Belelovsky K, Bar-Gad I (2009) The neurophysiological correlates of motor tics following focal striatal disinhibition. Brain 132:2125-2138.

Meana JJ, Johansson B, Herrera-Marschitz M, O’Connor WT, Goiny M, Parkinson FE, Fredholm BB, Ungerstedt U (1992) Effect of the neurotoxin AF64A on intrinsic and extrinsic neuronal systems of rat neostriatum measured by in vivo microdialysis. Brain Res 596:65-72.

Planert H, Szydlowski SN, Hjorth JJ, Grillner S, Silberberg G (2010) Dynamics of synaptic transmission between fast-spiking interneurons and striatal projection neurons of the direct and indirect pathways. J Neurosci 30:3499-3507.

Ponzi A, Wickens J (2010) Sequentially switching cell assemblies in random inhibitory networks of spiking neurons in the striatum. J Neurosci 30:5894-5911.

Samoilova MV, Buldakova SL, Vorobjev VS, Sharonova IN, Magazanik LG (1999) The open channel blocking drug, IEM-1460, reveals functionally distinct alpha-amino-3-hydroxy-5-methyl-4-isoxazolepropionate receptors in rat brain neurons. Neuroscience 94:261-268.

Schildein S, Huston JP, Schwarting RK (2002) Open field habituation learning is improved by nicotine and attenuated by mecamylamine administered posttrial into the nucleus accumbens. Neurobiol Learn Mem 77:277-290.

Shirley TL, Rao LM, Hess EJ, Jinnah HA (2008) Paroxysmal dyskinesias in mice. Mov Disord 23:259-264.

Taverna S, Ilijic E, Surmeier DJ (2008) Recurrent collateral connections of striatal medium spiny neurons are disrupted in models of Parkinson's disease. J Neurosci 28:5504-5512.

Wilson CJ (2007) GABAergic inhibition in the neostriatum. Prog Brain Res 160:91-110

Wiltschko AB, Gage GJ, Berke JD (2008) Wavelet filtering before spike detection preserves waveform shape and enhances single-unit discrimination. J Neurosci Methods 173:34-40.

Wiltschko AB, Pettibone JR, Berke JD (2010) Opposite effects of stimulant and antipsychotic drugs on striatal fast-spiking interneurons. Neuropsychopharmacology 35:1261-1270.

Witten IB, Lin SC, Brodsky M, Prakash R, Diester I, Anikeeva P, Gradinaru V, Ramakrishnan C, Deisseroth K (2010) Cholinergic interneurons control local circuit activity and cocaine conditioning. Science 330:1677-1681.

Worbe Y, Baup N, Grabli D, Chaigneau M, Mounayar S, McCairn K, Féger J, Tremblay L (2009) Behavioral and movement disorders induced by local inhibitory dysfunction in primate striatum. Cereb Cortex 19:1844-1856.

Zhou LW, Zhang SP, Connell TA, Weiss B (1993) Cholinergic lesions of mouse striatum induced by AF64A alter D2 dopaminergic behavior and reduce $\mathrm{D} 2$ dopamine receptors and D2 dopamine receptor mRNA. Neurochem Int 22:301-311. 